\title{
Primary pulmonary lymphangiectasia in Noonan syndrome: apropos of an extremely rare manifestation and a brief literature review
}

\author{
Georgia-Emmanuela Dendrinou ${ }^{1}$, Panagiotis Zagarelos ${ }^{2}$, Angelos Sofronas ${ }^{3}$, Stamatis Katsenos ${ }^{1}$ \\ ${ }^{1}$ Department of Pneumonology; ${ }^{2}$ Department of Endocrinology; ${ }^{3}$ Department of Radiology, 401 General Military \\ Hospital of Athens, Greece
}

\begin{abstract}
Noonan syndrome (NS) is a genetic multisystem disorder characterised by distinctive facial features, developmental delay, learning difficulties, short stature, congenital heart disease, renal anomalies, bleeding difficulties and lymphatic malformations. Although lymphatic dysplasias are present in $20 \%$ of patients with NS, however pulmonary lymphangiectasia has rarely been described. In this present paper, we report a 24 -year-old male who was diagnosed with Noonan syndrome and primary pulmonary lymphangiectasia by using chest imaging modalities. A brief overview of the current literature is also provided laying emphasis on the clinical, pathogenetic and diagnostic aspects of this uncommon Noonan syndrome complication.
\end{abstract}

Correspondence: Stamatis Katsenos, Department of Pneumonology, 401 General Military Hospital of Athens, 158 Mesogion \& Katehaki Avenue, 11525 Athens, Greece.

E-mail:skatsenos@yahoo.gr

Key words: Noonan syndrome; pulmonary lymphangiectasia.

Contributions: GED was responsible for patient management and prepared the manuscript; PZ helped to confirm Noonan syndrome and draft the manuscript; AS performed and interpreted the imaging modalities results; SK critically revised the manuscript and approved the final version. All authors have read and approved the final manuscript and agreed to be accountable for all aspects of the work.

Conflict of interest: The authors declare that they have no competing interests, and all authors confirm accuracy.

Consent for publication: The patient gave his written consent to use his personal data for the publication of this case report and any accompanying images.

Funding: The study was not supported by any specific finding.

Received for publication: 18 August 2020.

Accepted for publication: 23 September 2020.

${ }^{\circ}$ Copyright: the Author(s), 2020

Licensee PAGEPress, Italy

Monaldi Archives for Chest Disease 2020; 90:1576

doi: 10.4081/monaldi.2020.1576

This article is distributed under the terms of the Creative Commons Attribution Noncommercial License (by-nc 4.0) which permits any noncommercial use, distribution, and reproduction in any medium, provided the original author(s) and source are credited.

\section{Introduction}

Noonan syndrome is an autosomal dominant disorder with an estimated prevalence of 1 in 1000-2500 [1]. This genetic disease is classified within the "RASopathies", a family of related developmental syndromes caused by upregulation of RAS-MAPK signalling pathway [2]. As a consequence of RAS-MAPK pathway derangement, Noonan syndrome is a multisystem disease mostly characterized by distinctive facial features, short stature, congenital heart disease, renal and bleeding abnormalities, developmental delay, as well as lymphatic malformations [3]. Although lymphatic dysplasias are present in $20 \%$ of patients with NS, however pulmonary lymphangiectasia has rarely been described. Herein, we report a well-documented case of Noonan syndrome with associated primary pulmonary lymphangiectasia and include a short review regarding clinical, pathogenetic and diagnostic aspects of this unusual entity.

\section{Case Report}

A 24-year-old male, non-smoker, was referred to our department for further evaluation of an abnormal result on a chest radiograph obtained as part of the health assessment for his military service. His medical history was unremarkable, except for hypothyroidism and episodes of otitis media. However, a thorough clinical examination revealed short stature, pectus excavatum and several distinctive facial features including hypertelorism (wide spaced eyes), epicanthal folds and exophthalmos of the right eye, downward slanting palpebral fissures, micrognathia, low set posteriorly rotated ears with a thick helix and short neck with prominent nuchal skin (Figure $1 \mathrm{a}-\mathrm{d}$ ). These findings were typical of Noonan syndrome, according to the criteria set by van der Burgt and outlined in Table 1 [4].

Further work-up was carried out to investigate organ defects relevant to the Noonan syndrome. In particular, an echocardiogram demonstrated moderate aortic and mild mitral valve regurgitation. An audiological examination revealed a mild hearing loss due to three episodes of otitis media in his early childhood. Both gonadal function and blood coagulation screening tests were normal. Bone mineral density (BMD) z-score using dual-energy Xray absorptiometry (DXA) of lumbar spine was significantly reduced at -2.6 thus indicating osteoporosis. Although the aforementioned characteristic external ocular features, a complementary ophthalmological examination disclosed amblyopia of the right eye as well as left eye exophoria. Last but not least, his chest x-ray was abnormal showing bilateral reticular interstitial markings (Figure 2). Further imaging evaluation with high-resolution com- 
puted chest tomography disclosed parenchymal inhomogeneity with patchy areas of ground-glass infiltrates predominantly in perihilar and subpleural regions as well as thickening of the interlobular septa, suggestive of lymphatic channel disturbance (Figure 3 a-c). Pulmonary function tests (spirometry, static lung volumes measurement) and lungs diffusing capacity were normal. Fibreoptic bronchoscopy was performed revealing a normal whole tracheobronchial tree. Bronchoalveolar lavage (BAL) samples for respiratory pathogens identification and cytological examination as well as BAL cell differential counts did not demonstrate abnormal findings. Despite the patient refusal to undergo an open lung biopsy, the diagnosis of primary pulmonary lymphangiectasia due to Noonan syndrome was made, based on the characteristic clinical features and distinctive HRCT findings. Therefore, the patient was recommended to undergo an annual follow-up by a multidisciplinary medical team including cardiologist, endocrinologist, ophthalmologist and pneumonologist. Moreover, a great deal of attention should be paid to avoid respiratory infections.

\section{Discussion}

Congenital pulmonary lymphangiectasia (PL) is a rare developmental disorder involving the lung and is characterized by pulmonary subpleural, interlobar, perivascular, and peribronchial lymphatic dilatation. Its incidence is estimated at approximately $1 \%$ in consecutive necropsies on stillbirths and neonates [5]. The true incidence after the newborn period is unknown and reports of pulmonary lymphangiectasia in infants and children are restricted to isolated case reports. According to the improved characterization of the clinical presentation and recent remarkable advances in intensive neonatal care, the condition is divided into two major categories, defined as primary and secondary pulmonary lymphangiectasia [6,7]. Primary form encompasses developmental abnormalities that are either generalized (part of a general lymphangiectasia involving various organ systems), pulmonary (confined to the lungs) or syndromic (associated with unrelated congenital abnormalities) whereas secondary form comprises those abnormalities due to lymphatic or cardiovascular obstruction, or acquired through other means (such as infection) [8].

Pulmonary lymphangiectasia may present at birth as a stillbirth or with severe respiratory distress, tachypnoea, and cyanosis thus exhibiting a very high mortality rate at or within a few hours of birth [9]. The disease can be strongly suspected in full-term neonates who present severe respiratory distress with pleural and pericardial effusion or ascites (especially if chylous) at birth, with or without generalized or localized lymphedema. In the postneonatal period, children with PL present with respiratory difficulties of varying degree, associated with a relapsing course. In older children it is commonly associated with recurrent cough, wheeze, increased respiratory effort with inspiratory crackles, and even congestive heart failure. The disease is characterized by frequent respiratory exacerbations $[5,8]$.

Table 1. Diagnosing criteria for Noonan syndrome.

\begin{tabular}{|c|c|c|}
\hline Feature & Major criteria & Minor criteria \\
\hline Facial & $\begin{array}{l}\text { Typical face dysmorphology (Hypertelorism, broad forehead, ptosis, epicanthic } \\
\text { folds and downward slanting palpebral fissures, low-set posteriorly rotated ears with } \\
\text { a thick helix, high arched palate, micrognathia, short broad neck with excessive nuchal } \\
\text { skin and low posterior hairline) }\end{array}$ & Suggestive face dysmorphology \\
\hline Cardiac & $\begin{array}{l}\text { Pulmonary valve stenosis, HOCM, and/or ECG typical of NS (wide QRS with negative } \\
\text { pattern in left precordial leads and left axis deviation with giant Q waves) }\end{array}$ & Other defect \\
\hline Height* & $<3^{\text {rd }}$ percentile for age & $<10^{\text {th }}$ percentile for age \\
\hline Chest wall & Pectus carinatum/excavatum & Broad thorax \\
\hline Family history & First degree relative with definite NS & First degree relative with suggestive NS \\
\hline Other & Mental retardation, cryptorchidism, and lymphatic dysplasia (all three present) & $\begin{array}{l}\text { Mental retardation, cryptorchidism, or lymphatic } \\
\text { dysplasia (one of three present) }\end{array}$ \\
\hline
\end{tabular}

HOCM, hypertrophic obstructive cardiomyopathy; ECG, electrocardiogram; NS, Noonan syndrome; *normal range for height is considered here to be between the $3^{\text {rd }}$ and $97^{\text {th }}$ percentile. A definitive diagnosis of NS can be made in two scenarios: (1) Major facial features present plus one other major or two minor criteria; (2) Minor facial features present plus two major or three other minor criteria.
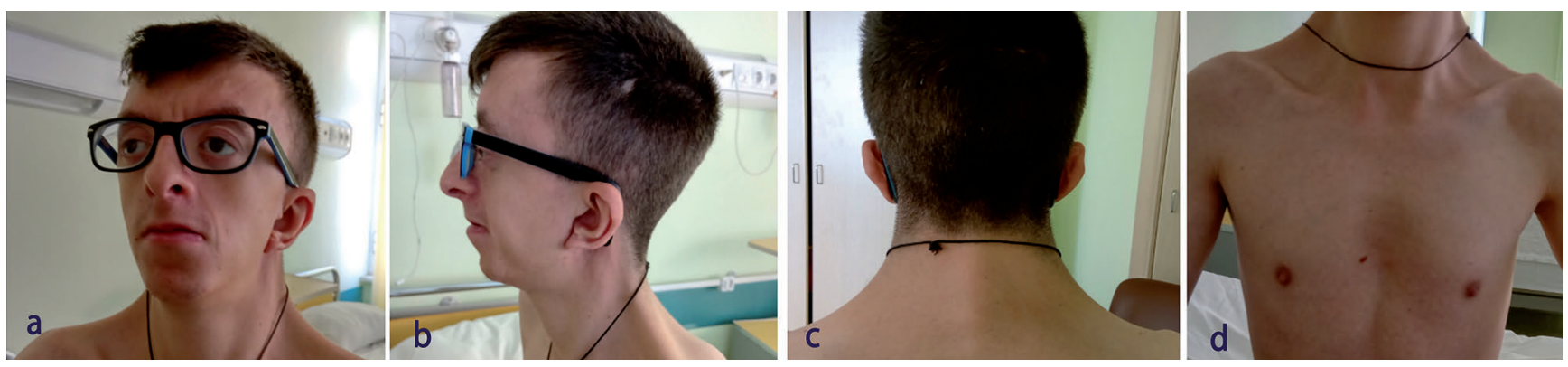

Figure 1. a) Distinctive facial features of Noonan syndrome: hypertelorism, epicanthal folds and exophthalmos of the right eye, downward slanting palpebral fissures. b) Low set posteriorly rotated ears with a thick helix. c) Short neck with prominent nuchal skin. d). Pectus excavatum. 
It is now generally suggested that primary pulmonary lymphangiectasia is a result of a failure of normal regression process of the pulmonary lymphatics after the $16^{\text {th }}$ week of gestation thus leading to persistence of the large subpleural, interlobar, perivascular and peribronchial lymphatic vessels that are normal form of the maturation developmental process at 9-16 weeks of gestation [10]. Mutations in specific genes, such as forkhead box C2 (FOXC2) and SRY (sex determining region Y) box 18(SOX18), which are both involved in the lymphatic vasculature development, have been implicated in the causation of pulmonary lymphangiectasia [11]. In particular, Noonan syndrome is a relatively common genetic multisystem disease that causes several congenital abnormalities, including lymphatic malformations, albeit infrequent ones. This genetic disorder is caused by mutations in genes involved in RAS-MAPK signalling pathway, an essential mediator of developmental processes. The RAS-MAPK pathway transduces signals that instruct the cell to grow and divide, to differentiate, to migrate and to survive, thus regulating several vital cellular processes. This regulation tightly controls the growth of cells and tissues and has been rendered indispensable for proper development. Therefore, mutations in genes encoding proteins involved in RAS-MAPK pathway cause dysregulation of this pathway with detrimental effects on both embryonic and postpartum development. PTPN11 gene mutations account for approximately $50 \%$ of all cases of Noonan syndrome [1-3]. The PTPN11 gene encodes SHP-2, a cytoplasmic protein tyrosine phosphatase (PTP), which is a critical component of signal transduction for several growth factor-, hormone-, and cytokine-signalling pathways controlling developmental processes, haematopoiesis/lymphopoiesis as well as energy balance and metabolism [12,13]. PTPN11 variants have been reported in patients with Noonan syndrome and associated bilateral chylothoraces, intestinal and pulmonary lymphangiectasia, genital oedema and cardiac abnormalities in a recently published study [14].

Diagnostic techniques that may be useful in evaluating pulmonary lymphangiectasia include conventional radiologic studies, high-resolution computed tomography (HRCT) and MR imaging, lymphoscintigraphy, lymphangiography, bronchoscopy and surgical lung biopsy [6]. Chest radiograph usually shows hyperinflation

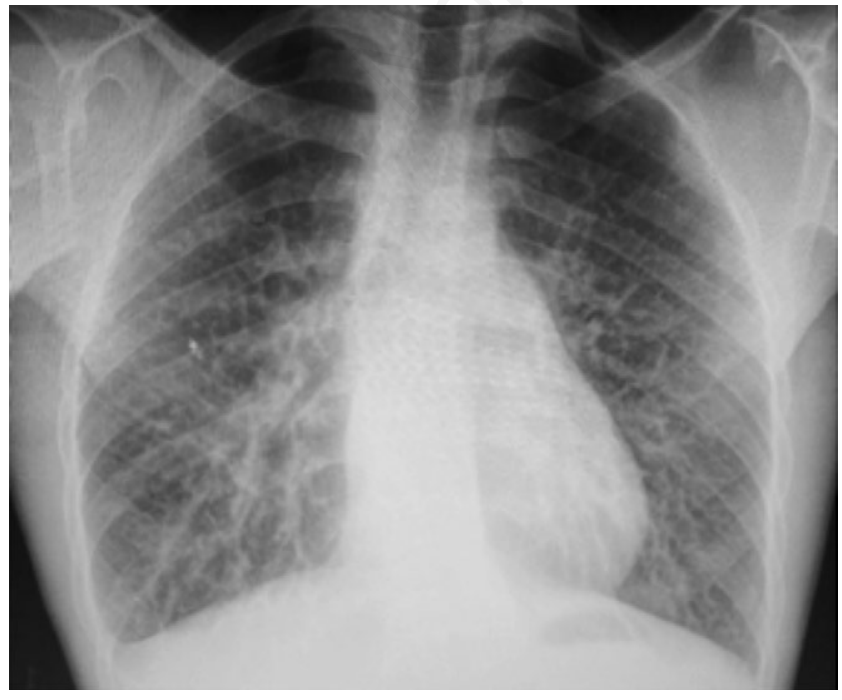

Figure 2. Chest radiograph showing bilateral reticular interstitial markings. with interstitial markings HRCT demonstrates diffuse thickening of the interstitium, both of the peribronchovascular interstitium and the septa surrounding the lobules, as well as ground glass
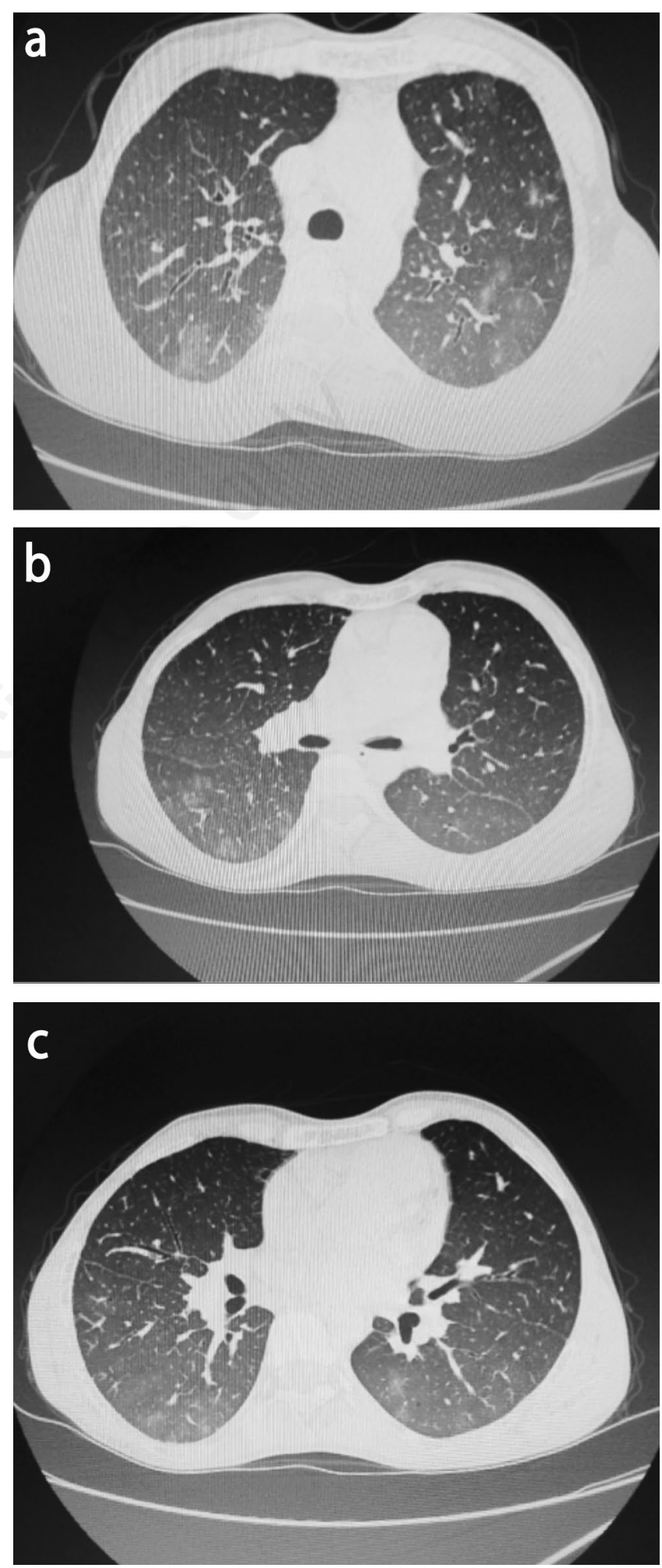

Figure 3. Chest high resolution computed tomographies demonstrating sparse ground glass opacities accompanied by thickening of the interlobular septa. 
opacities and pleural effusion. It is considered to be one of the most accurate diagnostic tests for CPL [15]. Similar findings have been noted in post-natal MRI. Nevertheless, HRCT is preferable to MRI not only in diagnosing PL, but also for the identification of paediatric interstitial lung disease. Lymphoscintigraphy is another useful imaging modality in evaluating the morphological and functional status of the lymphatic system. In particular, it can detect lung lymph vessel involvement by showing radiotracer accumulation in the lungs and by providing evidence of back-flow within the thoracic duct $[9,14]$. Conventional lymphangiography or dynamiccontrast MR lymphangiography have also been used as imaging lymphatic techniques in patients with Noonan syndrome and central lymphatic abnormalities. Retrograde intercostal lymphatic flow, pulmonary lymphatic perfusion, and agenesis or dysgenesis of the central lymphatic conduction system are the main characteristic findings [16]. Although bronchoscopic examination is not specifically indicated in PL, it may be helpful for ruling out other pulmonary entities and performing bronchoalveolar lavage to isolate respiratory pathogenic organisms. No tracheobronchial anatomical abnormalities were reported in PL patients who were evaluated by bronchoscopy. Lung biopsy may be beneficial in PL diagnosis demonstrating the presence of dilated lymphatic spaces in the sub-pleural connective tissue, along the thickened interlobar septa, and around the bronchovascular axes [17]. However, close attention must be paid when preparing histological specimens and interpreting lung biopsies or autopsy samples. In fact, the pathological findings in PL patients may change over time, especially in case of viral infection, and, more generally, may vary from initial recognition of minimal evidence of lymphatic dilatation, possibly related to a technical artifact (cross-clamping of the lung), to confirmation of severe lymphangiectasia.

Contradictory data have been reported pertaining to the prognosis of primary pulmonary lymphangiectasia. Earlier case studies have demonstrated dismal prognosis with mortality rate approaching $100 \%$ before the $1990 \mathrm{~s}$ in neonatal period and childhood $[8,18]$. Nonetheless, the outcome seems to be favourable, according to recent case series and retrospective studies [5,19]. The survival improvement might be due to advances in neonatal intensive care therapy and also to the fact that severity can vary among affected children. It has been suggested that outstanding survival rates can be anticipated in neonatal onset primary pulmonary lymphangiectasia without additional anomalies/comorbidities as well as for the postneonatal onset form. Moreover, a small case series study found that children with Noonan syndrome and the localized forms of PPL have a better chance of long-term survival than do children with involvement of both lungs and without a diagnosis of Noonan syndrome [20]. As far as the present case is concerned, it can be deduced from the clinical picture, imaging and pulmonary function testing results that the disease runs an indolent course. More specifically, the patient did not mention any severe respiratory system symptomatology, had normal lung function tests and exhibited limited extent of pulmonary lesions on chest CT imaging.

In conclusion, primary pulmonary lymphangiectasia is an uncommon developmental disease mostly occurring in neonatal period and early childhood. It is very rarely observed in adulthood. Symptomatology is generally present despite its occasional accidental discovery in an asymptomatic adult. HRCT scanning is an instrumental imaging modality in establishing the diagnosis of primary pulmonary lymphangiectasia. When the diagnosis is made in childhood or adult age, the clinical outcome is more likely to be promising, albeit recent improvements in intensive neonatal care have changed the previously nearly fatal outcome of PPL at birth.

\section{References}

1. Roberts AE, Allanson JE, Tartaglia M, Gelb BD. Noonan syndrome. Lancet 2013;381:333-42.

2. Tartaglia M, Zampino G, Gelb BD. Noonan syndrome: Clinical aspects and molecular pathogenesis. Mol Syndromol 2010;1:2-26.

3. Tafazoli A, Eshraghi P, Koleti ZK, Abbaszadegan M. Noonan syndrome - a new survey. Arch Med Sci 2017;13:215-22.

4. van der Burgt I. Noonan syndrome. Orphanet J Rare Dis 2007;2:4-10.

5. Barker PM, Esther CR Jr, Fordham LA, et al. Primary pulmonary lymphangiectasia in infancy and childhood. Eur Respir J 2004;24:413-9.

6. Bellini C, Boccardo F, Campisi C, Bonioli E. Congenital pulmonary lymphangiectasia. Orphanet J Rare Dis 2006;1:43.

7. Doecke EA. Congenital pulmonary lymphangiectasia. J Lymphoedema 2014;9:30-5.

8. Esther CR Jr, Barker PM. Pulmonary lymphangiectasia: Diagnosis and clinical course. Pediatr Pulmonol 2004;38:308-13.

9. Bellini C, Mazzella M, Campisi C, et al. Multimodal imaging in the congenital pulmonary lymphangiectasia-congenital chylothorax-hydrops fetalis continuum. Lymphology 2004;37:22-30.

10. Mele P, Sridhar S. Congenital pulmonary lymphangiectasia: an unusual presentation of nonimmune hydrops in a preterm infant. Adv Neonatal Care 2012;12:166-71.

11. deBruyn G, Casaer A, Devolder K, et al. Hydrops fetalis and pulmonary lymphangiectasia due to FOXC2 mutation: an autosomal dominant hereditary lymphedema syndrome with variable expression. Eur J Pediatr 2012;171:447-50.

12. Qu CK, Nguyen S, Chen J, Feng GS. Requirement of Shp-2 tyrosine phosphatase in lymphoid and hematopoietic cell development. Blood 2001;97:911-4.

13. Mathur D, Somashekar S, Navarrete C, Rodriguez MM. Twin infant with lymphatic dysplasia diagnosed with Noonan syndrome by molecular genetic testing. Fetal Pediatr Pathol 2014;33:253-7.

14. Joyce S, Gordon K, Brice G, et al. The lymphatic phenotype in Noonan and Cardiofaciocutaneous syndrome. Eur J Hum Genet 2016;24:690-6.

15. Nobre LF, Muller NL, de Souza Junior AS, et al. Congenital pulmonary lymphangiectasia: CT and pathologic findings. J Thorac Imaging 2004; 19:56-9.

16. Biko DM, Reisen B, Otero HJ, et al. Imaging of central lymphatic abnormalities in Noonan syndrome. Pediatr Radiol 2019;49:586-92.

17. Reiterer F, Grossauer K, Morris N, et al. Congenital pulmonary lymphangiectasis. Paediatr Respir Rev 2014;15:275-80.

18. Faul JL, Berry GJ, Colby TV, et al. Thoracic lymphangiomas, lymphangiectasis, lymphangiomatosis, and lymphatic dysplasia syndrome. Am J Respir Crit Care Med 2000;161:1037-46.

19. Mettauer N, Agrawai S, Pierce C, et al. Outcome of children with pulmonary lymphangiectasis. Pediatr Pulmonol 2009; 44:351-7.

20. Chung CJ, Fordham LA, Barker P, Cooper LL. Children with congenital pulmonary lymphangiectasia: after infancy. AJR Am J Roentgenol 1999;173:1583-8. 